EUROPEAN ORGANIZATION FOR NUCLEAR RESEARCH

European Laboratory for Particle Physics

LHC Project Report 21

Large Hadron Collider Project

\title{
Comparison of Floating and Thermalized Multilayer Insulation Systems at Low Boundary Temperature
}

\author{
Gerard Ferlin, Berthold Jenninger, Philippe Lebrun, Guillermo Peon, \\ Germana Riddone and Balazs Szeless
}

\begin{abstract}
The Large Hadron Collider (LHC) will be a $26.7 \mathrm{~km}$ circumference particle collider using high-field superconducting magnets operating in superfluid helium. An efficient and robust thermal insulation system is therefore required to minimise the residual heat inleak to the large surface area at $1.9 \mathrm{~K}$ constituted by the stainless steel wall of the helium enclosure. The baseline solution uses "floating" multilayer reflective insulation. An alternative consists of a combination of multilayer reflective films and a soft screen, partially thermalized to the $5 \mathrm{~K}$ level and supported away from the cold wall by net-type insulating spacers. We establish the improvement potential of the alternative over the baseline solution, and compare their insulation performance on the basis of measured characteristics of thermal contacts and spacers.
\end{abstract}

LHC Division, CERN, CH-1211 Geneva 23, Switzerland

ICEC 16/ICMC, Kitakyushu, Japan, 20-24 May 1996

Administrative Secretariat

LHC Division

CERN

CH - 1211 Geneva 23

Switzerland

Geneva, 12 July 1996 
Gerard Ferlin, Berthold Jenninger, Philippe Lebrun, Guillermo Peon, Germana Riddone and Balazs Szeless

\title{
LHC Division, CERN, CH-1211 Geneva, Switzerland
}

\begin{abstract}
The Large Hadron Collider (LHC) will be a $26.7 \mathrm{~km}$ circumference particle collider using high-field superconducting magnets operating in superfluid helium. An efficient and robust thermal insulation system is therefore required to minimise the residual heat inleak to the large surface area at $1.9 \mathrm{~K}$ constituted by the stainless steel wall of the helium enclosure. The baseline solution uses "floating" multilayer reflective insulation. An alternative consists of a combination of multilayer reflective films and a soft screen, partially thermalized to the $5 \mathrm{~K}$ level and supported away from the cold wall by net-type insulating spacers. We establish the improvement potential of the alternative over the baseline solution, and compare their insulation performance on the basis of measured characteristics of thermal contacts and spacers.
\end{abstract}

\section{INTRODUCTION}

Large cryogenic projects, such as superconducting particle accelerators and colliders, expose large areas of cold surface to residual heat flux from the ambient-temperature environment, and therefore demand efficient and robust thermal insulation systems. This is particularly true of the Large Hadron Collider (LHC), presently under construction at CERN [1], with 1600 high-field superconducting magnets operating in superfluid helium [2] distributed around the $26.7 \mathrm{~km}$ circumference of the machine tunnel. The residual heat inleak from the $75 \mathrm{~K}$ thermal shield, which reaches the $50^{\prime} 000 \mathrm{~m}^{2}$ of surface area at $1.9 \mathrm{~K}$ presented by the stainless steel wall of the helium enclosure, represents the single largest contribution to the thermal budget of the cryostats [3]. This was identified early in the project, and an experimental program initiated, to investigate and qualify thermal insulation systems at low boundary temperature on representative samples and geometries. First results [4] confirmed the advantage of multilayer systems over single reflective surfaces for coping with degraded insulation vacuum, a situation bound to happen locally over the circumference of the machine, and further tests [5] sought to improve the performance of "floating" multilayer systems by limiting parasitic conduction between layers. Cryostats for prototype cryomagnets [6] and a full-size thermal model [7] were built in industry and operated with such an insulation system, bringing a real-scale verification of the thermal performance measured on samples and thus validating thermal budget estimates for the LHC [8]. In view of the importance of this source of heat inleak to the 1.9 $\mathrm{K}$ level, we investigate in the following the potential of improvement presented by an alternative combining multilayer reflective films and a soft screen, actively thermalized to the $5 \mathrm{~K}$ level and supported away from the cold wall by net-type insulating spacers. After establishing the range of thermal impedances of interest for thermal contacts [9] to the $5 \mathrm{~K}$ pipe, and insulating spacers [10], we show how these can be realised in practical systems, and present results of measurements on test samples.

\section{POTENTIAL OF A THERMALIZED MULTILAYER INSULATION SYSTEM}

Figures 1 and 2 show the principle and the thermal flow scheme for floating and thermalized multilayer superinsulation systems. Heat flux through multilayer insulation is given by the combination of solid conduction, residual gas conduction and radiation. 


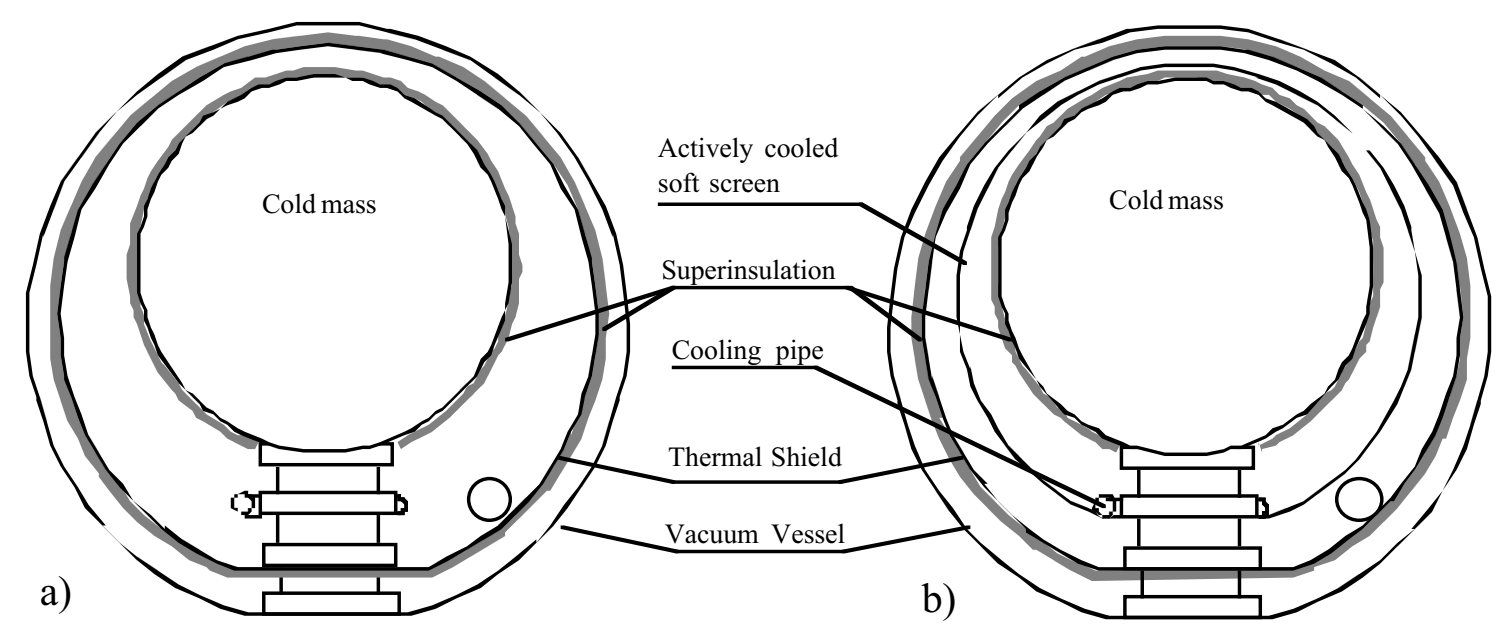

Figure 1: Principle scheme of (a) floating and (b) thermalized multilayer systems

In the thermalized case the actively cooled soft screen receives heat $\left(\mathrm{Q}_{\mathrm{ss}}\right)$ from the thermal shield by solid conduction (SC1), residual gas conduction $(\mathrm{GC} 1)$ and radiation $(\mathrm{R} 1)$. From the soft screen heat flows to the thermalization pipe (at $5 \mathrm{~K}$ ) by solid conduction $\left(\mathrm{Q}_{\mathrm{cp}}\right)$ and to the cold mass $\left(\mathrm{Q}_{\mathrm{cm}}\right)$ by solid conduction through the spacers (SC2), residual gas conduction (GC2) and radiation (R2). Solid conduction, residual gas conduction and radiation are expressed respectively by Fourier's law, Kennard's law and Boltzmann's law. Heat inleak at $1.9 \mathrm{~K}$ depends on the soft screen temperature and on the thermal impedance between the soft screen and the cold mass:

$$
\mathrm{Q}_{\mathrm{cm}}=\frac{\mathrm{T} 1-\mathrm{T} 2}{\mathrm{R}_{\mathrm{cm}}} \quad \text { with } \quad \mathrm{R}_{\mathrm{cm}}=\frac{\mathrm{r}_{\mathrm{cm}}}{\mathrm{As}} \quad \mathrm{Q}_{\mathrm{cp}}=\frac{\mathrm{T} 1-\mathrm{T} 3}{\mathrm{R}_{\mathrm{cp}}}
$$

Low residual heat flux to the cold mass can be attained if the thermal contact between the screen and the cooling pipe performs well $\left(\mathrm{R}_{\mathrm{cp}}\right.$ small) and if the cold mass is well insulated from the screen $\left(\mathrm{r}_{\mathrm{cm}}\right.$ large).

a)

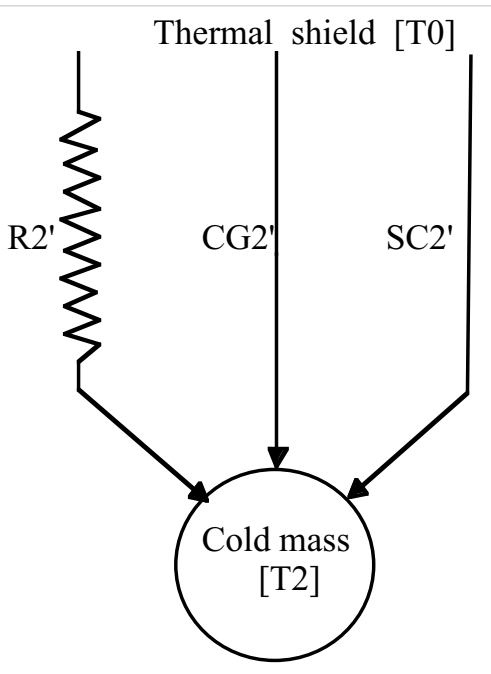

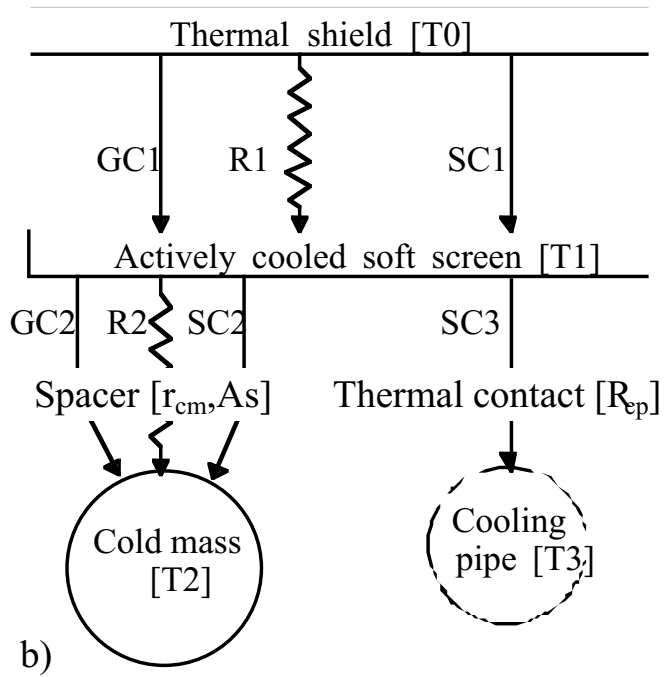

Heat transfer processes

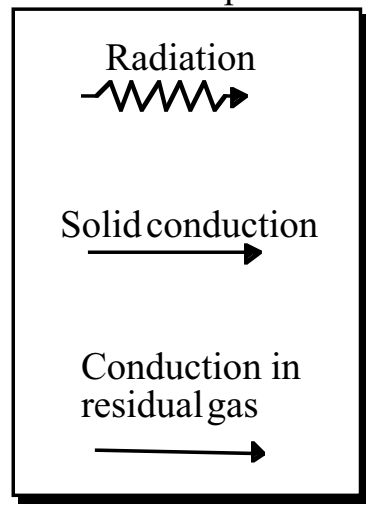

Figure 2: Thermal flow-scheme of (a) floating and (b) thermalized multilayer systems

Applying this model to a 15-m long LHC dipole cryostat, we can find the range of validity of values of $\mathrm{R}_{\mathrm{cp}}$ and $\mathrm{r}_{\mathrm{cm}}$ for which a thermalized system performs better than floating multilayer insulation. Figure 3 shows the break-even lines comparing with the 10-layer system of type B in reference [4], which presently equips the LHC prototype cryomagnets [6-8], assuming an emissivity of the soft screen of 0.05 in the 5 to $30 \mathrm{~K}$ range. The surface covered by the spacers has been considered equivalent to $25 \%$ of the total cold mass area. The thermalized version has lower heat inleak at $1.9 \mathrm{~K}$ if the design point of the two thermal impedances, $\mathrm{r}_{\mathrm{cm}}$ and $\mathrm{R}_{\mathrm{cp}}$, is placed in the region above the break-even lines. 


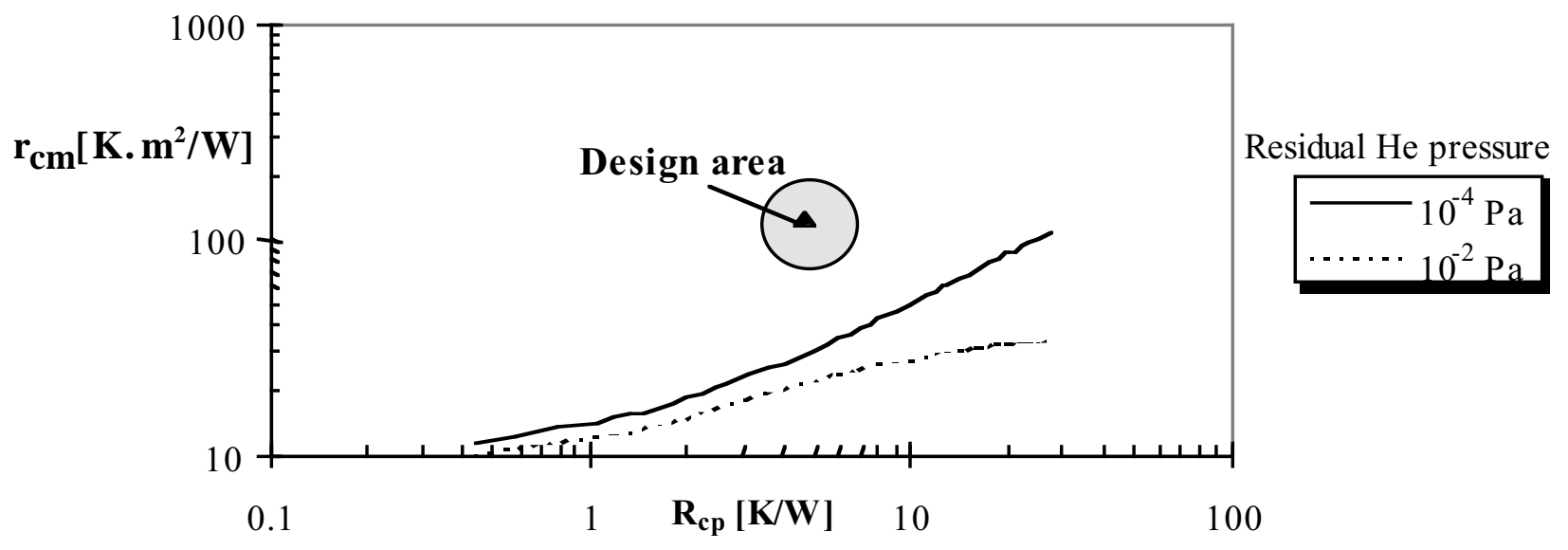

Figure 3: Break-even lines for thermalized versus floating systems, in terms of $\mathrm{R}_{\mathrm{cp}}$ and $\mathrm{r}_{\mathrm{cm}}$

\section{THERMAL CONTACT TO COOLING PIPE}

A dedicated experiment (figure 4) has been performed to measure the impedance of thermal contacts on a geometry, materials and assembly techniques of industrial nature, representative of heat intercepts to be implemented in large numbers in LHC cryostats. The heat intercept is constituted of a conductive sleeve, made of aluminium, shrink-fitted onto a DN65 stainless steel tube and connected to an isothermally heated block by means of aluminium strips. The thermal impedance between the aluminium sleeve and the stainless steel tube is evaluated from temperature measurements with a precision of $\pm 10 \%$. The temperature difference shows, as expected, a linear dependence on the heat flow $\mathrm{Q}$. The thermal contact between the aluminium sleeve and the stainless steel tube has been tested at different temperatures and performs as follows: $1.2 \pm 0.1 \mathrm{~K} / \mathrm{W}$ at $4.5 \mathrm{~K}$ and $0.5 \pm 0.05 \mathrm{~K} / \mathrm{W}$ at $20 \mathrm{~K}$. Samples with different diameters and materials will be tested in order to investigate the consequence on thermal impedance.

\section{NET-TYPE INSULATING SPACERS}

The insulating spacers considered consist of a set of piled-up nets of non-metallic, low-thermal conductivity materials which provide support at low residual heat flux. Different type of nets are alternatively inserted to avoid large contact area due to accidental superposition. A specific experiment (figure 4) has been developed to measure, by means of a heatmeter [11], the heat flux between two circular aluminium plates separated by spacers. The cold plate temperature is at $2 \mathrm{~K}$ and that of the warm plate, which simulates the soft screen, can be varied from $10 \mathrm{~K}$ to $30 \mathrm{~K}$. The compressive force can be increased from $11 \mathrm{~N}$ to $94 \mathrm{~N}$. The spacers are made of a combination of two types of glass fiber nets (N1 and N2) from industry. N1 has a 10x10 net grid with a thread thickness of $1 \mathrm{~mm}$. N2 has a $6 \times 6$ net grid with a thread thickness of $0.5 \mathrm{~mm}$. A spacer composed of 3 layers of $\mathrm{N} 1$ and 2 layers of N2 have been tested. For a warm temperature of $25 \mathrm{~K}$ and with a compressive force of $34 \mathrm{~N}$, the measured heat flux to $2 \mathrm{~K}$ at $10^{-4} \mathrm{~Pa}$ is $10 \pm 1 \mathrm{~mW}$, which gives a thermal impedance of about $70 \mathrm{~K} \cdot \mathrm{m}^{2} / \mathrm{W}$.

\section{COMPARATIVE PERFORMANCE}

Considering a thermal contact resistance of $5 \mathrm{~K} / \mathrm{W}$ (contact over $30 \%$ of length of soft screen at about $10 \mathrm{~K}$ ) and an insulator spacer thermal impedance of $100 \mathrm{~K} \cdot \mathrm{m}^{2} / \mathrm{W}$ at $10^{-4} \mathrm{~Pa}$ we can calculate the variation of heat inleak at $1.9 \mathrm{~K}$ as a function of residual gas pressure and compare it to the performance of the reference solution. The change of thermal resistance between soft screen and cold mass with insulation vacuum has been evaluated. Figure 5 shows that the thermalized version has lower residual heat flux both for nominal and degraded vacuum. At $10^{-4} \mathrm{~Pa}$ the residual heat flux to the cold mass as low as $30 \mathrm{~mW} / \mathrm{m} 2$ can in principle be achieved.

An actively cooled screen will be mounted in a full-scale thermal model $[7,8]$ and heat inleak measurements will be carried out in order to confirm sample measurements and predicted performance. 


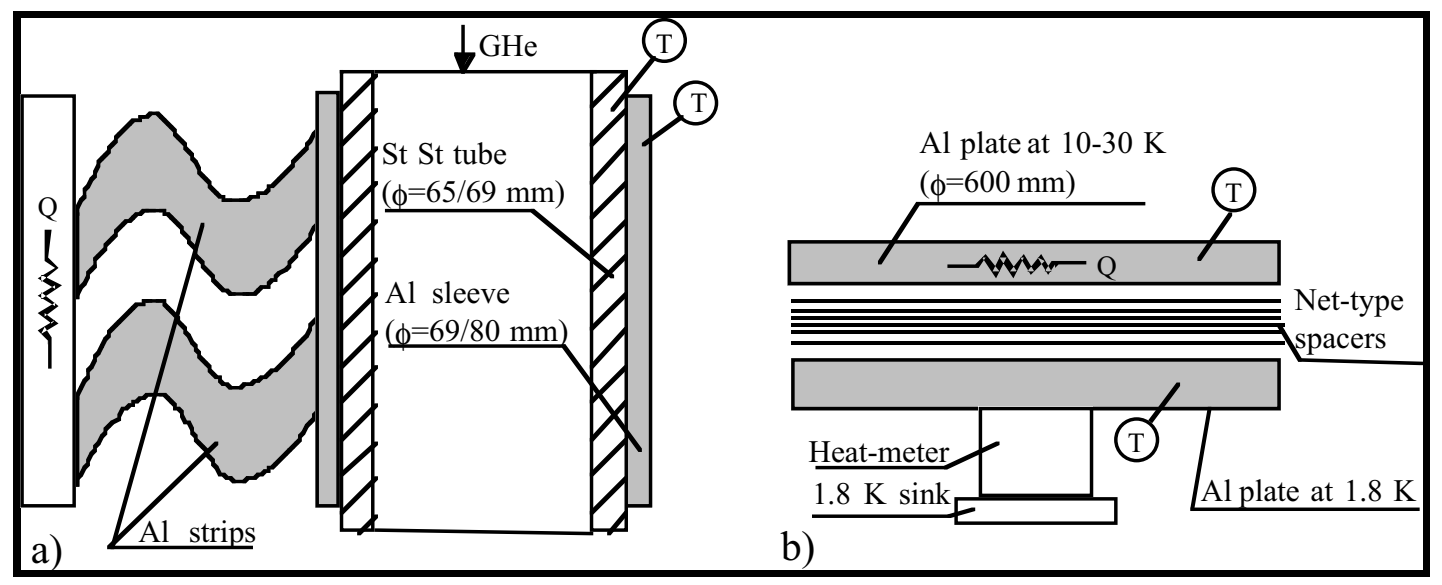

Figure 4: Experimental measurements of thermal impedance of thermal contacts (a) and net-type insulating spacers (b) under variable applied force

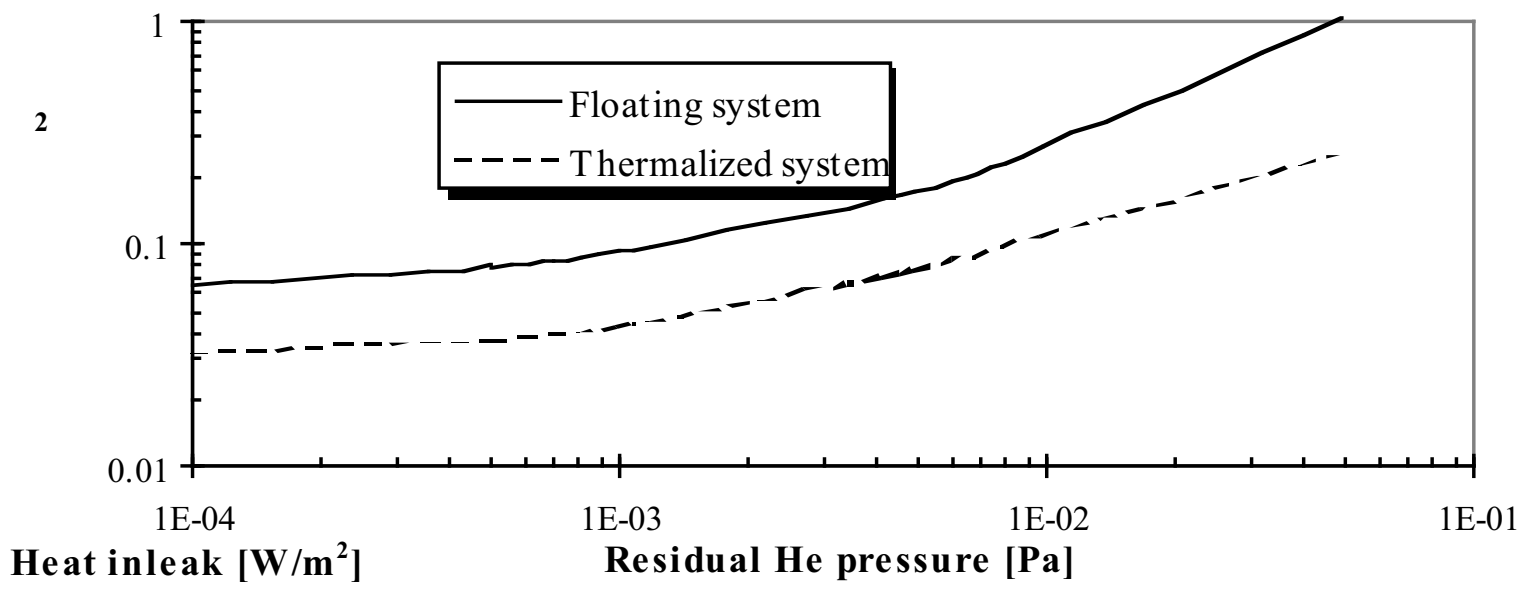

Figure 5: Comparison of thermal performance between floating and thermalized systems.

\section{REFERENCES}

1 Evans, L.R., The Large Hadron Collider project, presented at this conference.

2 Lebrun, Ph., Superfluid helium cryogenics for the Large Hadron Collider project at CERN, Cryogenics (1994) 34 ICEC Supplement 1-8

3 The LHC Study Group, The Large Hadron Collider, Conceptual Design, Report CERN/AC/95-05 (LHC) (1995)

4 Lebrun, Ph., Mazzone, L., Sergo, V. and Vullierme, B., Investigation and qualification of thermal insulation systems between $80 \mathrm{~K}$ and 4.2 K, Cryogenics (1992) 32 ICMC Supplement 42-47

5 Benda, V., Lebrun, Ph., Mazzone, L., Sergo, V. and Vullierme, B., Qualification of multilayer insulation systems between $80 \mathrm{~K}$ and $4.2 \mathrm{~K}$, Proc. Kryogenika'94 Usti nad Labem (1994) 107-110

6 Brunet, J.C., Kerby, J., Lebrun, Ph., Rohmig, P., Szeless, B. and Williams, L., Design of LHC prototype dipole cryostats, Cryogenics (1992) 32 ICEC Supplement 191-194

7 Dufay, L., Ferlin, G., Lebrun, Ph., Riddone, G., Rieubland, J.M., Rijllart, A., Szeless, B. and Williams, L., A full-scale thermal model of a prototype dipole cryomagnet for the CERN LHC project, Cryogenics (1994) 34 ICEC Supplement 693-696

8 Benda, V., Dufay, L., Ferlin, G., Lebrun, Ph., Rieubland, J.M., Riddone, G., Szeless, B., Tavian, L. and Williams, L., Measurement and analysis of thermal performance of LHC prototype dipole cryostats, presented at CEC'95 Columbus (1995)

9 Riddone, G., Theoretical modelling and experimental investigation of the thermal performance of LHC lattice cryostats, Doctoral thesis, Politecnico di Torino, Italy (1996)

10 Peón, G., Thermo-mechanical study and optimisation of vapour shielded cryostats, Design of components of the half-cell LHC superfluid helium cryostat, Doctoral thesis (in preparation), University of Zaragoza, Spain

11 Danielsson, H., Lebrun, Ph. and Rieubland, J.M., Precision heat inleak measurements on cryogenic components at $80 \mathrm{~K}, 4.2 \mathrm{~K}$ and $1.8 \mathrm{~K}$, Cryogenics (1992) 32 ICEC Supplement 215-218 